\title{
NUEVOS REGISTROS DE ESCARABAJOS COPRÓFAGOS (COLEOPTERA: SCARABAEIDAE: SCARABAEINAE) PARA EL DEPARTAMENTO DE CHOCÓ (COLOMBIA)
}

Arias-Buriticá, J. A., P. Delgado-Gómez, F. A. González-A, F. Z. Vaz-de-Mello. 2011. New records of dung beetles (Coleoptera: Scarabaeidae: Scarabaeinae) for Chocó department (Colombia). Acta Zoológica Mexicana (n. s.), 27(3): 875-878.

ABSTRACT. We present nine new records for dung beetles (Coleoptera: Scarabaeidae: Scarabaeinae) for the department of Chocó (Colombia): Ateuchus aeneomicans, Canthon juvencus, C. cyanellus, Onthophagus coscineus, O. marginicollis, Sulcophanaeus miyashitai metallescens, Ateuchus candezei, Dichotomius favi and Uroxys platypyga also the last three being new records for Colombia, as well as expand the distribution of the same to South America.

Los escarabajos coprófagos (Scarabaeidae: Scarabaeinae) en Colombia están representados por 36 géneros con 289 especies (Medina et al. 2001; Pulido et al. 2007; Noriega et al. 2008). Sin embargo, el conocimiento preliminar de muchas zonas geográficas y el poco trabajo taxonómico realizado en el país hace que no se tenga certeza en la riqueza de especies del mismo.

Basados en los resultados de una evaluación rápida de diversidad (RAP) realizada en el departamento de Chocó, se encontraron nueve nuevos registros de especies, tres de ellos también nuevos para Colombia.

Las colectas se realizaron en enero de 2008 en el corregimiento de Capurganá, municipio de Acandí ubicado en el extremo norte del departamento de Chocó, noroccidente de Colombia. Capurganá presenta altitudes entre 20 y $200 \mathrm{~m}$, una temperatura media anual de $27^{\circ} \mathrm{C}$, precipitaciones de $2296 \mathrm{~mm}$ anuales con un régimen bimodal siendo los meses lluviosos de abril-agosto y octubre-noviembre, la vegetación dominante es el bosque húmedo tropical. Se seleccionaron tres bosques ubicados en los siguientes sitios: Jardín Botánico ( $8^{\circ} 37^{\prime} 42^{\prime}$ N, $77^{\circ} 21^{\prime} 12^{\prime}$ W; $30 \mathrm{~m}$ snm), Vía a Sapzurro ( $\left.8^{\circ} 38^{\prime} 23^{\prime \prime} \mathrm{N}, 77^{\circ} 21^{\prime} 16^{\prime \prime} \mathrm{W} ; 63 \mathrm{~m} \mathrm{snm}\right)$ y Bocatoma $\left(8^{\circ} 36^{\prime} 53^{\prime \prime} \mathrm{N}, 77^{\circ} 20^{\prime}\right.$ 
50"W; $198 \mathrm{~m} \mathrm{snm).} \mathrm{En} \mathrm{cada} \mathrm{sitio} \mathrm{de} \mathrm{muestreo} \mathrm{se} \mathrm{instaló} \mathrm{un} \mathrm{transecto} \mathrm{de} 15$ trampas de caída con excremento humano como atrayente, separadas $50 \mathrm{~m}$ de acuerdo con lo propuesto por Larsen \& Forsyth (2005) y dos trampas de intercepción de vuelo. Los dos métodos de captura estuvieron activos por 72 horas. Los individuos se encuentran depositados en las colecciones entomológicas de las siguientes instituciones: Museo de Historia Natural "Luis Gonzalo Andrade" de la Universidad Pedagógica y Tecnológica de Colombia (Tunja-Colombia); Instituto Alexander von Humboldt (Villa de Leyva-Colombia), Instituto de Ciencias Naturales, Universidad Nacional de Colombia (Bogotá-Colombia), Escarabajos Coprófagos de Colombia (Bogotá-Colombia) y Universidade Federal de Mato Grosso (Cuiabá-Brasil).

Ateuchus aeneomicans (Harold, 1868). Especie distribuida de Costa Rica a Colombia (Howden \& Young 1981; Kohlmann 1997), en el país ha sido registrada para el departamento de Caldas (Medina et al. 2001). Se capturaron dos individuos en trampa de intercepción de vuelo, en el punto de colecta vía a Sapzurro.

Ateuchus candezei (Harold, 1868). Especie registrada para Centroamérica desde México (Chiapas) hasta Panamá (Isla Barro Colorado) (Howden \& Young 1981; Kohlmann 1997). Este es el registro más al sur que se tiene de la especie (quitando antiguas citas dudosas para Brasil) ampliando su distribución en aproximadamente 280 $\mathrm{km}$. Se recolectaron 322 individuos en los tres sitios de muestreo y con los dos tipos de trampa utilizados.

Canthon cyanellus sallei Harold, 1863. Especie de distribución neotropical desde México hasta Venezuela con tres subespecies siendo C. cyanellus sallei la registrada para Colombia en los departamentos de Bolívar, Meta y Tolima (Medina et al. 2001). Se recolectaron 52 individuos en los sitios de muestreo Jardín Botánico y vía a Sapzurro, con los dos tipos de trampas utilizadas en el estudio. Además se observaron individuos recolectados en trampas con atún, que fueron utilizadas para la captura de hormigas (obs. pers.).

Canthon juvencus (Harold, 1868). Especie distribuida desde Panamá hasta Brasil (Howden \& Young 1981). Para Colombia ha sido registrada en seis departamentos (Bolívar, Guanía, Guaviare, Magdalena, Tolima y Meta) (Medina et al. 2001). Se recolectaron 79 individuos en los sitios de muestreo Jardín Botánico y vía a Sapzurro, en los dos tipos de trampas utilizadas en el estudio.

Dichotomius favi Kohlmann \& Solís, 1997. Especie conocida de Costa Rica (Kohlmann \& Solís 1997). Este es el registro más al sur que se tiene de la especie ampliando su distribución en aproximadamente $600 \mathrm{~km}$. Se recolectaron 185 individuos 
en trampas con excremento humano en los sitios de muestreo Jardín Botánico y vía a Sapzurro.

Onthophagus coscineus Bates, 1887. Especie distribuida de Costa Rica a Colombia en donde se ha registrado para el departamento de Nariño (Medina et al. 2001). Se recolectaron 93 individuos en los tres sitios de muestreo y en los dos tipos de trampa utilizados en el estudio.

Onthophagus marginicollis Harold, 1880. Especie distribuida desde México hasta Brasil (Howden \& Young 1981). Para Colombia ha sido registrada en seis departamentos (Antioquia, Bolívar, Caldas, Cundinamarca, Tolima y Valle del Cauca) (Medina et al. 2001). Se recolectaron 8 individuos en los sitios de muestreo Jardín botánico y vía a Sapzurro, en trampas con excremento humano; se colectaron pocos individuos dado que esta especie está más adaptada a zonas abiertas (Howden \& Young 1981; Horgan 2001).

Sulcophanaeus miyashitai metallescens Arnaud, 2002. Subespecie descrita con ejemplares del departamento de Valle del Cauca (Arnaud 2002). Este es el registro más al norte que se tiene de la subespecie ampliando su distribución en aproximadamente $550 \mathrm{~km}$. Se capturó una hembra en el sitio La Bocatoma en trampa con excremento humano. El primer autor capturó machos y hembras de esta subespecie en muestreos realizados en zonas cercanas a Quibdó, en el centro del departamento de Chocó.

Uroxys platypyga Howden \& Young, 1981. La especie se encuentra distribuida desde México (Chiapas) (Navarrete \& Halffter 2008) hasta Panamá (Isla de Barro Colorado) (Howden \& Young 1981), este es el registro más al sur que se tiene de la especie ampliando su distribución en $280 \mathrm{~km}$. Se recolectaron 155 individuos en los tres sitios de muestreo y en los dos tipos de trampa utilizados en el estudio.

\section{LITERATURA CITADA}

Arnaud, P. 2002. Les coléoptères du monde vol. 28: Phanaeini. Hillside Books, Canterbury.

Horgan, F. 2001. Burial of bovine dung by coprophagous beetles (Coleoptera: Scarabaeidae) from horse and cow grazing sites in El Salvador. European Journal of Soil Biology, 37: 103-111.

Howden, H. \& O. Young. 1981. Panamanian Scarabaeinae Taxonomie distribution and habits Contributions. American Entomological Institute, 18: 1-204.

Kohlmann, B. 1997. The Costa Rican species of Ateuchus (Coleoptera: Scarabaeidae). Biología Tropical, 44/45: 177-192.

Kohlmann, B. \& A. Solís. 1997. El género Dichotomius (Coleoptera: Scarabaeidae) en Costa Rica. Giornale Italiano di Entomologia, 8: 343-382.

Larsen, T. \& A. Forsyth. 2005. Trap spacing and transect design for dung beetle biodiversity studies. Biotropica, 37: 322-325. 
Medina, C., A. Lopera-Toro, A. Vítolo, \& B. Gill. 2001. Escarabajos Coprófagos (Coleoptera: Scarabaeidae: Scarabaeinae) de Colombia. Biota Colombiana, 2: 131-144.

Navarrete, D. \& G. Halffter. 2008. Nuevos registros de escarabajos copro-necrófagos (Coleoptera: Scarabaeidae: Scarabaeinae) para México y Chiapas. Acta Zoológica Mexicana, 24: 247-250.

Noriega, J., J. Rengifo \& F. Vaz de Mello. 2008. First report of the genus Tetramereia Klages, 1907 (Coleoptera: Scarabaeidae: Phanaeini) in Colombia - notes to its distribution. Biota Colombiana, 9: 131-133.

Pulido, L., C. Medina \& R. Riveros. 2007. Nuevos registros de escarabajos coprófagos (Scarabaeidae: Scarabaeinae) para la región andina de Colombia. Revista de la Academia Colombia de Ciencias Físicas y Exactas, 31: 305-310.

\section{Jorge Armando Arias-Buriticá, ${ }^{1,2}$ Paola Delgado-Gómez, ${ }^{1,3}$ Fabio Arturo GoNZÁLEZ-A $^{1,4}$ y Fernando Zagury VAZ-DE-MELLo ${ }^{5}$}

${ }^{1}$ Universidad Pedagógica y Tecnológica de Colombia.

Grupo de Investigación Sistemática Biológica (SisBio).

Avenida Central del Norte,

Escuela de Ciencias Biológicas,

Tunja (Boyacá-Colombia).

$2<$ georgebiology@gmail.com>

$3<$ padego@gmail.com>

$4<$ fagakorn@gmail.com>

${ }^{5}$ Universidade Federal de Mato Grosso.

Instituto de Biociências,

Departamento de Biologia e Zoologia.

Av. Fernando Correa da Costa,

2367 Boa Esperança.

<vazdemello@gmail.com> 